PSICOLOGI A SOCIAL

\title{
Estilos de Aprendizagem em EaD: Construção e evidências de validade de instrumento
}

\section{Learning Styles in DE context: Construction and evidences of instrument validity}

\section{Estilos de aprendizaje en EAD: Construcción y evidencias de validez del instrumento}

\author{
Raíssa Bárbara Nunes Moraes* \\ Universidade de São Paulo - USP, Ribeirão Preto, São Paulo, Brasil \\ Thaís Zerbini** \\ Universidade de São Paulo - USP, Ribeirão Preto, São Paulo, Brasil
}

\begin{abstract}
RESUMO
A expansão de projetos de educação a distância (EAD) e seus consequentes programas de treinamento, formação e qualificação profissionais ofertados por instituições de ensino superior (IES) e outras organizações, tornam necessário um maior número estudos sistemáticos nesse contexto. Destacase a necessidade de estudos relacionados às variáveis do perfil da clientela, como é o caso dos Estilos de Aprendizagem. A presente pesquisa objetivou construir e verificar evidências de validade de um instrumento de medida de Estilos de Aprendizagem no contexto de EAD em IES. Participaram do estudo, virtualmente, alunos de uma universidade pública no interior paulista matriculados em um curso superior a distância. Foram realizadas análises fatoriais exploratórias e de consistência interna. A escala possui três fatores: Ambiente de Estudo ( 2 itens, $a=0,76$ ); Regulação Externa (4 itens, $a=0,63$ ); Interação Interpessoal ( 2 itens, $a=0,40$ ). Por ser um instrumento inédito na área da Psicologia Organizacional e do Trabalho (POT) no contexto de EAD em IES, são necessários mais estudos, bem como aplicação em amostras diferentes.
\end{abstract}

Palavras-chave: aprendizagem, educação à distância, validação de instrumento.

\section{ABSTRACT}

The expansion of distance education (DE) projects and its training and qualification programs offered by higher education institutions (HEIs) and organizations creates the need of a higher number of systematic studies in this field. It's possible to emphasize the need for studies related to the customer profile variables as the Learning Styles. This research is focused in building and verifying evidences of the validity of a Learning Styles measuring instrument in the DE and HIEs context. Students enrolled in a higher education course in distance education of a public university from a city in the state of São Paulo attended virtually to this research. Exploratory factorial and intern consistency analysis were made in this study. The scale 
has three factors: Study Environment ( 2 items, $a=0,76)$; External Regulation (4 items, $a=0,63$ ); Interpersonal Interation ( 2 items, $a=0,40$ ). This is a unpublished instrument in the Organization and Work Psychology in the DE and HIEs context. Therefore, it's requires more studies and apply it in different samples.

Keywords: learning styles, distance education, instrument validation.

\begin{abstract}
RESUMEN
La expansión de los proyectos de educación a distancia (EAD) y sus programas de formación y capacitación que ofrecen las instituciones de educación superior (IES) y las organizaciones crea la necesidad de un mayor número de estudios sistemáticos en este campo. Es necesario realizar estudios relacionados con las variables del perfil del cliente como los estilos de aprendizaje. Esta investigación se centra en la construcción y la verificación de evidencias de la validez de un instrumento de medición de Estilos de Aprendizaje en el contexto EAD y IES. Los estudiantes inscritos en un curso de educación superior en la educación a distancia de una universidad en el estado de Sao Paulo asistieron virtualmente a esta investigación. El análisis factorial y la consistencia pasante exploratoria se hizo en este estudio. La escala tiene tres factores: ambiente de estudio (2 artículos, $a=0,76)$; La regulación externa (4 artículos, $a=0,63) ; \mathrm{La}$ interacción interpersonal ( 2 artículos, $a=0,40)$. Se requiere de más estudios que pueden incluir o modificar algunos artículos y aplicarlo en diferentes muestras.
\end{abstract}

Palabras clave: aprendizaje, educación a distancia, validación del instrumento.

\title{
1 I ntrodução
}

Os programas de Treinamento, Desenvolvimento e Educação (TD\&E) despertam o interesse científico pelo modo como vêm sendo oferecidos, uma vez que se constituem instrumentais de expressivo valor para ações de formação educacional favorecedoras da aquisição de competências vitais e valorizadas pela sociedade atual (Coelho Jr. \& Borges-Andrade, 2008). Frente a cenários altamente voláteis, os eventos instrucionais à distância tornam-se uma estratégia para instituições de ensino superior (IES) e organizações laborais, já que propiciam o estabelecimento dos mecanismos responsáveis pela aprendizagem efetiva e otimização de desempenhos. Experimentando um crescimento vertiginoso, a Educação a Distância (EAD) atesta suas inegáveis contribuições aos processos formativos ao se estabelecer como iniciativa educacional que permite servir a um quinhão de indivíduos cada vez maior, diminuir custos e otimizar o tempo (Coll, Mauri \& Onrubia, 2010; Varanda, Zerbini \& Abbad, 2010; Zerbini \& Abbad, 2008).

A EAD enfrenta sérios desafios envolvendo fatores que interferem sobre a eficiência e empregabilidade de tais esforços instrucionais. Uma das mais relevantes problemáticas existentes no campo da Educação e do Treinamento a Distância é a escassa produção e 
sistematização de conhecimentos acerca das causas que interferem sobre o avanço e conclusão das ações formais de ensinoaprendizagem não presenciais (Abbad, Carvalho \& Zerbini, 2006; Sales, 2009; Walter, 2006).

Uma das características da EAD é a de ser adaptável uma vez que é planejada, normalmente, para ser usada por uma grande variedade de indivíduos com diferentes habilidades, preferências ou Estilos de Aprendizagem (Brusilovsky \& Peylo, 2003). As investigações sobre os Estilos de Aprendizagem fornecem informações para 0 aperfeiçoamento dos métodos instrucionais e para mudanças de comportamento dos que aprendem (Bjork, Dunlosky \& Kornell, 2013; Vermunt, 2005).

No tocante as razões que levam o aluno a não concluir um programa de ensino a distância, a literatura indica motivos provenientes das características dos estudantes (Estilos de aprendizagem, Estratégias de aprendizagem, Autoeficácia, entre outras), das restrições inerentes aos contextos de estudo do participante e do desenho e execução do curso (Sales, 2009). Nessa publicação foi investigado um dos aspectos referentes às características do público-alvo (Estilos de Aprendizagem).

Para investigar melhor a lacuna referente aos Estilos de Aprendizagem, foi realizado um levantamento bibliográfico nas bases de dados consideradas importantes em revisões de TD\&E (Martins, 2012). Assim, buscou-se por resultados de pesquisas em Estilos de Aprendizagem em cursos de ensino superior ofertados à distância. Para a atualização e sistematização de resultados de pesquisa sobre as variáveis de interesse do presente estudo, foram realizadas revisões bibliográficas em bases de dados eletrônicas, abrangendo o período de 2007 a 2014. Tal período se justifica no sentido de que a produção que embasou o estudo sobre Estilos de Aprendizagem na área de Psicologia Organizacional e do Trabalho no Brasil foi publicada em 2007.

Foram analisadas produções científicas nacionais e internacionais na biblioteca eletrônica da CAPES, nas bases de dados ProQuest e Web of Science, além dos bancos de dissertações e teses da Universidade de São Paulo (USP) e da Universidade de Brasília (UnB), por serem bases apontadas em estudos anteriores (Martins, 2012; Umekawa \& Zerbini; 2015) com grande número de publicações na área da Psicologia Organizacional e do Trabalho. O levantamento bibliográfico visou examinar os avanços na construção e validação de instrumentos nacionais e estrangeiros que se dedicaram a mensurar os Estilos de Aprendizagem em alunos de cursos superiores a distância. Para tal, foram usadas as palavras chaves Estilos de Aprendizagem (Learning Styles), Educação a distância (Distance Education), Ensino Superior (Higher Education) e e-learning. 
Para uma maior adequação à pesquisa, foram selecionadas produções das áreas de Psicologia e da Educação. A segunda se justifica pela alta produção sobre Estilos de Aprendizagem. A revisão da literatura encontrou o total de 1226 trabalhos. Dentre eles foram selecionados os trabalhos das áreas de Psicologia e Educação, resultando em 23 artigos, dos quais 10 estavam disponíveis para download.

Dos trabalhos lidos, quatro são de origem nacional e os demais foram publicados na Turquia, Austrália, Estados Unidos e Inglaterra. Os instrumentos utilizados nos artigos foram, em primeiro lugar, o ILS Índice de Estilos de Aprendizagem (Felder \& Silverman, 1988), utilizado em três estudos; em segundo lugar, o LSI - Inventário de Estilos de Aprendizagem (Kolb, 1985; Kolb \& Kolb, 2005), utilizado em dois estudos; e em terceiro lugar o Questionário de Estilos de Aprendizagem (Honey \& Mumford, 2000), utilizado em um estudo. Os demais estudos eram de origem teórica, ou tinham como objetivo a construção e validação de uma medida de Estilos de Aprendizagem. Em relação às medidas citadas acima, somente o Questionário de Estilos de Aprendizagem foi validado no estudo, pois era esse 0 objetivo do mesmo. Os demais foram utilizados nos estudos, mas não foram submetidos à verificação de evidências de validade. Cabe destacar também que somente os estudos do Brasil tinham como objetivo a construção e validação de instrumento.

Em relação às áreas do conhecimento, seis artigos são da área da Educação e quatro da Psicologia, sendo que dentre esses quatro, dois são da Psicologia Educacional e dois da Psicologia Organizacional e do Trabalho. Disso, podemos concluir que a produção científica de Estilos de Aprendizagem na área da Psicologia está escassa.

Para Vermunt (1998), Estilos de Aprendizagem consistem em um conjunto coerente de atividades de aprendizagem que os indivíduos utilizam frequentemente, de acordo com a orientação e as concepções individuais de aprendizagem. Esse autor apresentou um modelo teórico de Estilos de Aprendizagem, o Modelo de Regulação dos Processos de Aprendizagem, cuja abordagem integra as noções de aprendizagem puramente cognitivista e a construcionista, e é composto por quatro dimensões hipotéticas de aprendizagem: a) estratégias de processamento de informação (atividades de pensamento que os alunos usam para processar conteúdos de aprendizagem); b) estratégias regulatórias de aprendizagem (atividades direcionadas para a regulação das atividades cognitivas e, portanto, levam a resultados de aprendizagem indiretamente); c) modelo mental de aprendizagem (conjunto coerente de concepções acerca da própria aprendizagem) e d) orientações para a aprendizagem (domínio de objetivos pessoais, intenções, motivos, expectativas, atitudes, preocupações e dúvidas dos alunos na realização de cursos ou estudos). 
Berings et al. (2005) definem os Estilos de Aprendizagem como uma tendência a usar uma determinada combinação de atividades para aprender que deriva de uma combinação de preferências e capacidades percebidas. Esse modelo teórico foi desenvolvido especificamente para o estudo de Estilos de Aprendizagem no contexto do trabalho e recebeu o nome de Modelo de Interação de Comportamento de Aprendizagem no Trabalho. Segundo ele, os Estilos de Aprendizagem são uma tendência a usar uma combinação particular de atividades implícitas e explícitas (ligadas a fatores individuais e ambientais, respectivamente) que uma pessoa pode e gosta de desempenhar. Tais atividades de aprendizagem, nomeadas mentais ou expostas, realizadas de maneira individual (intrapessoal) ou com outras pessoas (interpessoal), definem as dimensões dos Estilos de Aprendizagem.

Para Warr e Allan (1998) e Zerbini (2007), Estilos de Aprendizagem são as preferências do estudante referentes aos aspectos contextuais e as maneiras de estudar; compreendem a preferência dos indivíduos por determinados comportamentos de estudo e por aspectos do contexto em que este ocorre. São preferências focadas no contexto e nas atividades de aprendizagem, tais como: nível de ruído e temperatura do ambiente ao estudar, horário de preferência de estudo, forma de estudo (individual, em grupo).

Salles (2007) observa que existe uma produção de pesquisas voltadas para o trabalho e para as organizações que abordam os Estilos de Aprendizagem, mas a minoria delas utiliza medidas desenvolvidas exclusivamente para o ambiente educacional. Brant (2014) afirma que há algumas pesquisas estrangeiras evidenciando a influência dos Estilos de Aprendizagem em resultados finais de treinamento à distância. Contudo, a análise da produção científica nacional em Estilos de Aprendizagem mostra que predominam fortemente os estudos na área educacional. As pesquisas voltadas para o contexto do trabalho e IES são raras e, em sua maioria, apresentam inconsistência na descrição do método e na apresentação de resultados. A autora aponta que não encontrou estudos que utilizaram as medidas disponíveis para a verificação das relações entre os Estilos de Aprendizagem e as características individuais e de contexto no campo específico da educação a distância, que é o campo de estudo desta pesquisa.

Praticamente todos os instrumentos possuem problemas relacionados a validade e fidedignidade. Em alguns outros, há algumas lacunas referentes à própria definição de Estilos de Aprendizagem. Nota-se uma fraca base teórica na maioria dos estudos, o que traz a consequente má elaboração das medidas (Brant, 2014). Por fim, novamente aparece a falta de pesquisas sobre o tema, o que dificulta o desenvolvimento e adaptação das medidas já existentes, bem como sua evolução. A autora afirma que, apesar do instrumento Index of 
Learning Styles ser pouco utilizado, é o que apresenta melhores evidências de validade, além de já ter sido utilizado no contexto educacional de ensino superior presencial e à distância. Esse último contexto é o selecionado para o presente trabalho. Sendo assim, tal instrumento foi selecionado como base para a construção do instrumento de Estilos de Aprendizagem em contexto de ensino superior ofertado a distância.

Levando em consideração a Psicologia Organizacional e do Trabalho, temos a produção de Salles (2007) e Brant (2014) que se aprofundam no estudo de Estilos de Aprendizagem, sendo, portanto, as principais referências desse estudo. Brant (2014) realizou uma revisão de literatura focada em Estilos de Aprendizagem. A partir disso, buscou evidências de validade desses instrumentos para auxiliar nos objetivos de sua tese, dentre eles a construção de um instrumento de Estilos de Aprendizagem no contexto do trabalho para ações educacionais ofertadas à distância.

\section{Método}

\subsection{Objetivo}

Construir e verificar evidências de validade do instrumento de Estilos de Aprendizagem em Ações Educacionais Ofertadas a Distância.

\subsection{Procedimento de construção do instrumento}

Para a construção de um instrumento, antes de tudo, é necessário ter uma definição do constructo que se pretende medir. Considerando que a presente pesquisa está situada na área de conhecimento da Psicologia Organizacional e do Trabalho, foi estabelecida a referência da área para a definição de Estilos de Aprendizagem. Sendo assim, temos a definição de Zerbini (2007), que diz: "São as preferências do estudante referentes aos aspectos contextuais e as maneiras de estudo. Compreendem a preferência dos indivíduos por determinadas Estratégias de Aprendizagem e por aspectos do contexto em que esta ocorre". Essa definição foi selecionada por tratar dos Estilos de Aprendizagem no contexto de ações educacionais em IES ofertadas à distância. Enquanto as autoras Salles (2007) e Brant (2014) se focaram no contexto do trabalho, presencialmente e a distância, respectivamente.

A partir disso, foi adotada uma abordagem qualitativa e realizada uma revisão de literatura que auxiliasse na criação dos itens. 0 levantamento bibliográfico visou examinar os avanços na construção e validação de instrumentos nacionais e estrangeiros que se dedicaram a mensurar os Estilos de Aprendizagem em alunos de 
cursos superiores a distância. Após realizada a revisão de literatura apresentada na introdução deste trabalho, foi selecionado o instrumento Index of Learning Styles (ILS) de Vermunt (1998), por ser um instrumento aplicado no contexto de IES em EaD com melhores índices de validade (Brant, 2014).

Este instrumento é composto por 120 itens e quatro domínios, a saber: Atividades de processamento da informação, com 27 itens e cargas fatoriais variando de 0,27 a 0,75; Atividades de regulação da aprendizagem, com 28 itens e cargas fatoriais variando de 0,67 a 0,82; Orientações de aprendizagem, 25 itens e cargas fatoriais variando de 0,35 a 0,72; Modelos mentais de aprendizagem, 40 itens e cargas fatoriais entre 0,24 e 0,84 . Os itens estão associados a uma escala tipo Likert de frequência de 5 pontos. O instrumento foi validado em uma Regular University (RU) em uma Open University (OU). A RU oferecia cursos de graduação presencialmente enquanto a OU oferecia cursos à distância (Vermunt, 1998). Tais índices se referem à validação estatística em uma OU e foram selecionados devido à semelhança com o objetivo do instrumento dessa pesquisa.

Por serem preferências do indivíduo, optou-se pela construção de uma escala de preferências, variando de "sempre prefiro" a "nunca prefiro", numa escala Likert de 5 pontos.

Foi realizada uma exclusão dos itens que segundo a Psicologia Organizacional e do Trabalho (POT) são relacionados às Estratégias de Aprendizagem, alterados alguns itens em sua redação, tornando a escala de preferência, e adicionados alguns itens que não foram contemplados por Vermunt e são parte constituinte da definição de Zerbini (2007). O cotejamento entre o instrumento e as definições supracitadas possibilitou a modificação, inclusão e exclusão de itens, tendo resultado em uma nova versão composta por 27 itens - mais adequados à aplicação em curso de ensino superior oferecido a distância.

\subsection{Procedimento de validação de semântica e por Juízes}

A versão final do conjunto de itens de Estilos de Aprendizagem obtida na revisão bibliográfica e na análise de conteúdo foi submetida à validação semântica, onde foram identificados e corrigidos os termos e nomenclaturas do instrumento, bem como a clareza dos itens e contará com participantes semelhantes à amostra desse estudo. Posteriormente, foi submetida à validação por juízes, na qual especialistas da área analisaram a representatividade, clareza e precisão dos itens, bem como avaliam as instruções e a adequação do instrumento produzido. Após essa validação, o instrumento está pronto para ser aplicado e, posteriormente, prosseguir a validação estatística do mesmo. 


\subsection{Procedimentos de validação estatística}

Todas as análises da validação dos instrumentos foram realizadas no SPSS versão 21.0. Foram realizadas análises exploratórias para identificação de casos omissos; casos extremos univariados e multivariados, multicolinearidade, singularidade e linearidade, de acordo com as orientações de Tabachnick e Fidell (2007).

Em seguida, iniciaram-se as análises fatoriais exploratórias do instrumento, seguindo as orientações de Pasquali (2004). A análise da matriz de covariância em termos de fatorabilidade e do número de fatores, foi realizada de acordo com a análise do tamanho das correlações e do teste de adequação da amostra de Kaiser-MeyerOlkin (KMO). A análise dos componentes principais (Principal Components - PC) foi realizada para obter a extração inicial dos fatores. Por outro lado, a análise fatorial (AF) analisou as covariâncias entre as variáveis, assumindo que as mesmas são produtos de uma causa comum entre elas: os fatores comuns.

Para analisar a estabilidade, foi verificado o quanto os itens eram bons representantes do fator pelo tamanho da carga fatorial. Para analisar a interpretabilidade, identificou-se o traço latente que é considerado a causa do agrupamento das variáveis, por meio da análise da literatura da área. Os escores fatoriais foram produzidos pela média dos escores das variáveis originais que pertencem ao fator.

\subsection{População e Amostra}

A Universidade de São Paulo - responsável pela oferta do curso a distância denominado "Licenciatura em Ciências" - estabeleceu parceria para a execução da presente pesquisa. $O$ curso "Licenciatura em Ciências", de feitio semipresencial, passou a ser ministrado pela USP visando satisfazer uma demanda de aperfeiçoamento nas etapas do Ensino Fundamental e Médio paulistas, e está incluso no Programa Universidade Virtual do Estado de São Paulo (UNIVESP). O curso destina-se ao preparo de indivíduos que desejam se tornar aptos à prática do magistério no Ensino Fundamental ou de docentes atuantes na Educação Básica.

De acordo com 0 site do curso partícipe (www.licenciaturaciencias.usp.br), todo ano são disponibilizadas 360 vagas anuais, que são distribuídas pelos 7 pólos. No pólo de Ribeirão Preto, soma-se aproximadamente 205 vagas, considerando os quatro anos de curso. Informações sobre Evasão não foram repassadas. Dessa maneira, não se pode afirmar com exatidão o número de inscritos no curso. Todos os alunos matriculados no curso foram convidados a participar da pesquisa e receberam os questionários para serem respondidos. A amostra foi composta por 135 
respondentes. Se considerarmos o total de 205 vagas, o índice de retorno foi de $65,8 \%$.

Os dados dos respondentes mostram que que a maioria dos alunos é do sexo feminino $(57,8 \%)$, casada $(48,9 \%)$ e com filhos $(58,5 \%)$. A maioria dos participantes tem entre 40 e 49 anos (30,4\%), possuindo, em média, 38 anos de idade ( $D P=10,16)$. A maioria dos alunos possui Ensino Superior Incompleto (38,5\%). Dentre as profissões apresentadas pelos participantes, a de professor(a), foi a mais frequente $(34,8)$. Por fim, a maioria afirmou ter experiência anterior no uso da internet $(94,8 \%)$.

Para os processos de Validação Semântica e por Juízes da escala de Estilos de Aprendizagem, participaram, respectivamente, dez estudantes de graduação com características semelhantes aos da amostra e pesquisadores mestres $(n=3)$ e doutor $(n=1)$. Para essa etapa, foram selecionados participantes que possuem conhecimento teórico ou prático na área de Psicologia Organizacional e do Trabalho.

\section{Resultados}

\subsection{Validação semântica e por juízes}

Abaixo são apresentados os resultados advindos da Validação Semântica e por Juízes do instrumento de Estilos de Aprendizagem. Todos os itens, de alguma forma, sofreram alteração. Para essa publicação, serão demonstradas apenas as discussões mais relevantes, que não se tratavam apenas de questões de escrita e semântica. Tais dados estão sistematizados na Tabela 1. Para facilitar a apresentação dos resultados, os itens serão agrupados de acordo com o tema que abordam. 
Tabela 1: Validação Semântica e Por Juízes do Instrumento de Estilos de Aprendizagem

\begin{tabular}{|c|c|c|c|}
\hline & Item proposto & Sugestões & Item final \\
\hline \multirow{3}{*}{ 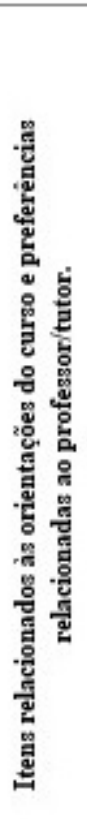 } & $\begin{array}{l}\text { 1) "...ser crítico em relação } \\
\text { ds interpretações de } \\
\text { especialistas", }\end{array}$ & $\begin{array}{l}\text { - Substituir "especialistas" por } \\
\text { "professores tutores" para uma } \\
\text { maior adequação ao contexto de } \\
\text { estudo. } \\
\text { - Inserção do item: "...ser crítico em } \\
\text { relação ao conteúdo apresentado } \\
\text { no material didático.", para } \\
\text { realizar uma contraposição a esse } \\
\text { item. }\end{array}$ & $\begin{array}{l}\text { "...ser crítico em relação } \\
\text { des discussões apresentadas } \\
\text { pelos professores/tutores" }\end{array}$ \\
\hline & $\begin{array}{l}\text { 16)"...que os instrutores } \\
\text { expliquem clavamente o que é } \\
\text { importante para que eu tome } \\
\text { conhecimento". }\end{array}$ & $\begin{array}{l}\text { - Adaptação semântica ao contexto } \\
\text { de estudo. } \\
\text { - Inserção do item: "...decidir } \\
\text { sosinho o que é importante } \\
\text { estudar para alcançar as objetivos } \\
\text { propostos no curso", tratando da } \\
\text { decisão do aluno em relação ao } \\
\text { que é mais importante estudar. }\end{array}$ & $\begin{array}{l}\text { "..que os } \\
\text { professores/tutores } \\
\text { expliquem claramente o } \\
\text { que é importante estudar } \\
\text { para alcançar os objetivas } \\
\text { propastos no curso" }\end{array}$ \\
\hline & $\begin{array}{l}\text { "...ser lembrado sobre as } \\
\text { prazos de entrega das } \\
\text { atividades do curso" }\end{array}$ & $\begin{array}{l}\text { - Incluido no instrumento após a } \\
\text { validação semântica } \\
\text { - Foi sugerida sua exclusão e } \\
\text { posterior condensação com o item } \\
15 \text {, que ficaria "...receber } \\
\text { informações detalhadas sobre os } \\
\text { prazos e as atividades a serem } \\
\text { realizadas no curso". }\end{array}$ & $\begin{array}{l}\text { Optou-se por permanecer } \\
\text { os dois itens, pois na } \\
\text { condensação o item } \\
\text { avaliaria dois constructos } \\
\text { (prazos e atividades), e } \\
\text { segundo Pasquali (1998) } \\
\text { um item deve avaliar } \\
\text { somente um constructo. }\end{array}$ \\
\hline \multirow{4}{*}{ 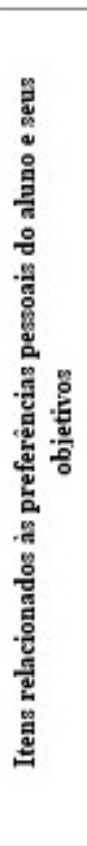 } & $\begin{array}{l}\text { 17) "...que tenham testes para } \\
\text { verificar se eu dominei todo o } \\
\text { assunto" }\end{array}$ & $\begin{array}{l}\text { - Melhoras na escrita para obter } \\
\text { uma maior clareza do item. } \\
\text { - Foi sugerido um item que } \\
\text { apresentasse uma contraposição, } \\
\text { sendo elaborado o enunciado: } \\
\text { "..avaliar sorinho meu domínio } \\
\text { do conteúdo apresentado" }\end{array}$ & $\begin{array}{lr}\text { "...que sejam realizadas } \\
\text { avaliaçóes r de } \\
\text { aprendizagem rara } \\
\text { verificar meu dominio do } \\
\text { conteúdo apresentado". }\end{array}$ \\
\hline & $\begin{array}{l}\text { "estudar no periodo da } \\
\text { noite", "estuday no periodo } \\
\text { da manhâ" e "estudar no } \\
\text { periodo da tarde" }\end{array}$ & $\begin{array}{l}\text { - Incluidos após a validação } \\
\text { semântica por serem preferências } \\
\text { importantes a serem medidas e foi } \\
\text { notada sua ausência. } \\
\text { - Na validação por juizes, foi } \\
\text { questionado sobre sua } \\
\text { permanência. }\end{array}$ & $\begin{array}{l}\text { Por serem três itens e pelo } \\
\text { fato que Zerbini (2007) } \\
\text { afirma sua importância } \\
\text { como um estilo de } \\
\text { aprendizagem e não um } \\
\text { dado demográfico, optou- } \\
\text { se pela permanência dos } \\
\text { mesmos. }\end{array}$ \\
\hline & $\begin{array}{l}\text { "..estabelecer metas de } \\
\text { desempenho no curso" }\end{array}$ & $\begin{array}{l}\text { - Incluido após a validação } \\
\text { semântica por ser considerado } \\
\text { uma preferência do aluno por } \\
\text { Brant (2014), foi questionado } \\
\text { sobre gua necessidade. }\end{array}$ & $\begin{array}{l}\text { O item foi excluido por } \\
\text { não ter sido encontra } \\
\text { nenhuma literatura que o } \\
\text { justificasge. }\end{array}$ \\
\hline & $\begin{array}{l}\text { "..tentar aprender sozinho } \\
\text { mesmo quando acho o } \\
\text { conteúdo dificil" }\end{array}$ & $\begin{array}{l}\text { - Incluido após validação semântica } \\
\text { por ser considerado uma } \\
\text { preferência do aluno. }\end{array}$ & $\begin{array}{l}\text { Excluido na validação } \\
\text { juizes por já ter sido } \\
\text { contemplado no item } \\
\text { "...realizar } \\
\text { individualmente } \\
\text { tarefas". }\end{array}$ \\
\hline
\end{tabular}


Tabela 1: Validação Semântica e Por Juizes do Instrumento de Estilos de Aprendizagem (continuação)

\begin{tabular}{|c|c|c|c|}
\hline \multirow{6}{*}{ 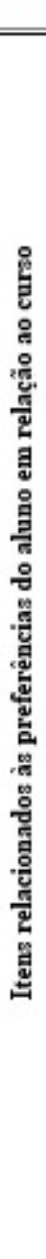 } & Item proposto & Sugestões & Item final \\
\hline & $\begin{array}{l}\text { 4) "-faser cursos que me } \\
\text { enviqueçam financeiramente" }\end{array}$ & $\begin{array}{l}\text { Questionado acerca da } \\
\text { impossibilidade de garantir } \\
\text { retorno financeiro após a } \\
\text { finalização do curso }\end{array}$ & Item excluido. \\
\hline & $\begin{array}{l}\text { 5) "...cursos que sejam um } \\
\text { passatempo para mim" } \\
\text { 6) "... facer qualquer curso, } \\
\text { pois gosto de estuda" } \\
\text { 12) "...cursos que sejam } \\
\text { adequados as minhas } \\
\text { capacidades de estudo" }\end{array}$ & - Falta de objetividade & Itens eliminados. \\
\hline & $\begin{array}{l}\text { 7) "... cursos que me } \\
\text { garantem un diploma" }\end{array}$ & $\begin{array}{l}\text { - Questionado acerca da redução } \\
\text { do ensino superior à obtenção de } \\
\text { um diploma, ignorando a } \\
\text { qualificação profissional, }\end{array}$ & $\begin{array}{l}\text { O item foi mantido devido } \\
\text { ao fato que atualmente o } \\
\text { diploma é muito } \\
\text { valorizado no mercado de } \\
\text { trabalho, formecendo um } \\
\text { maior numero de } \\
\text { oportunidades para os que } \\
\text { o possuem. Sendo asgim, } \\
\text { foi realizada somente uma } \\
\text { alteração semântica: "... } \\
\text { cuvsos que me garantem } \\
\text { um diploma/título". }\end{array}$ \\
\hline & $\begin{array}{l}\text { 10) "...cursas que me } \\
\text { possibilitem descobrir minhas } \\
\text { próprias qualidades es } \\
\text { limitą̧ões" }\end{array}$ & - Foi julgado como desnecessário. & $\begin{array}{l}\text { O item foi mantido com o } \\
\text { objetivo de medir } \\
\text { interesse do aluno em seu } \\
\text { autoconhecimento durante } \\
\text { o curso, sofrendo apenas } \\
\text { uma alteração semântica, } \\
\text { ficando: "t.cursos que } \\
\text { auxiliem a descoberta de } \\
\text { minhas qualidades } e \\
\text { limitações como } \\
\text { aprendis". }\end{array}$ \\
\hline & $\begin{array}{l}\text { 20) "...cursos que tenham } \\
\text { estratégias motivacionais } \\
\text { consistentes" }\end{array}$ & $\begin{array}{l}\text { - Questionado acerca do objetivo } \\
\text { do enunciado }\end{array}$ & $\begin{array}{l}\text { Item excluido por não estar } \\
\text { estruturado de maneira } \\
\text { clara a objetiva }\end{array}$ \\
\hline
\end{tabular}

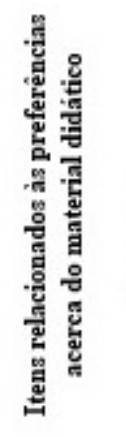

"...ler o material didático na tela do computador" e "ter o material impresso para ler $e$ faser anotaçöes"

- Incluidos após a validação semântica por serem considerados preferências dos alunos.

- Foram questionados na validação por juizes sob o argumento que um poderia eliminar o outro.
Optou-se por manter os dois itens, porem, foram reestruturados visando a avaliação apenas de um constructo em cada, ficando: "..fazer winhas anotações em arquivas digitais no computador. f."..ter o material impresso para ler".

A frase que orienta o preenchimento do instrumento, antes colocada como "para estudar em cursos de ensino superior à distância, eu prefiro..." não recebeu nenhuma crítica na validação semântica, porém, na validação por juízes, foi sugerido que se retirasse o "ensino superior", pois toda a amostra será composta por estudantes desse grau de escolaridade, além de diminuir a frase e torna-la mais dinâmica para a leitura e compreensão. Ficando, portanto, "para 
estudar em cursos à distância, eu prefiro...". As alterações propostas no processo de validação semântica e por juízes possibilitaram o aprimoramento e a adaptação da escala de medida em questão, que passou a ser constituída por 27 itens.

\subsection{Validação estatística}

Antes de iniciar a explanação acerca dos resultados das análises exploratórias do instrumento de Estilos de Aprendizagem, é necessário descrever o percurso que levou a esses resultados. Em um primeiro momento, foram realizadas todas as análises necessárias (linearidade, multicolinearidade, casos omissos, casos extremos e Principal Components - PC). Após realizar a análise paralela de Horn, obteve-se 4 fatores e realizou-se a Principal Axis Factoring - PAF). No momento do cálculo da consistência interna (a), um dos fatores obteve um valor negativo nessa variável. O Alfa de Cronbach deve ser interpretado no intervalo entre 0 e 1 , onde os valores negativos do alfa devem ser considerados como escalas sem confiança (ou seja, zero) (Nichols, 1999). Considerando isso, realizou-se novamente a PAF com três e dois fatores, sempre com o mesmo problema no momento de verificação da consistência interna. Uma observação importante é que o instrumento era fatorizável, uma vez que o índice de KMO estava acima de 0,70. Além dos valores negativos do Alfa, muitos itens possuíam cargas fatoriais e comunalidades extremamente baixas. Sendo assim, fez-se necessário a revisão dos itens, pois os cálculos estavam indicando um possível erro de medida. Tal revisão foi realizada e foram eliminados os itens que não estavam contemplados na definição de Estilos de Aprendizagem de Zerbini (2007) e Warr e Allan (1998) - definições usadas como referência para este trabalho por serem pertencentes à Psicologia Organizacional e do Trabalho, ou que ainda estavam contemplados em outras definições, como por exemplo Estratégias de Aprendizagem.

A revisão do instrumento resultou num total de 13 itens. Porém, mais uma vez obteve-se problemas nas análises exploratórias. Os itens 5) "...estudar no período da noite", 11) "...estudar no período da manhã" e 19) "...estudar no período da tarde" estavam se agrupando e formando um fator, e independente dos rearranjos que pudessem serem feitos (retirada de um dos três itens, visto que dois não poderiam ser retirados simultaneamente, uma vez que não existe fator de um único item), o valor do Alfa desse fator se apresentava negativo. Buscou-se então, literatura que justificasse a inclusão dessas preferências como um Estilo de Aprendizagem, e foi encontrado apenas em Zerbini (2007). Por questões estatísticas, foi optado pela retirada dos três itens. Em uma futura pesquisa, aconselha-se a inserção dos mesmos novamente, pois pode ser uma 
característica dessa amostra não possuir preferências em relação ao horário de estudo, visto que a grande maioria trabalha e não tem opções de horário para realizar as atividades acadêmicas, dispondo somente do tempo no qual não está no emprego. Sendo assim, o questionário final ficou com 10 itens, sendo eles: 4, 10, 12, 13, 15, $20,21,23,25$ e 26. Feitas todas essas considerações, serão apresentados aqui os resultados encontrados após as revisões e releituras do instrumento.

O questionário de Estilos de Aprendizagem apresentou 135 casos válidos e entre 0 e 1 um caso omisso nos seus 10 itens, com exceção do item 13 que apresentou 2 casos omissos. Dadas as recomendações de Tabachnick e Fidell (2007), quando o total de casos omissos não ultrapassa $5 \%$ dos dados, não é preciso realizar nenhuma análise ou alteração dos dados. As respostas apresentaram 8 casos extremos univariados, distribuídos entre 3 dos 10 itens, cerca de 2 ou 3 por item. Por ser também um número muito baixo, optouse por mantê-los. Não há consequências que influenciam negativamente nos resultados, pois, segundo Pasquali (2004), a AFE é robusta para casos extremos. Não foram identificados casos extremos multivariados.

A matriz de covariância foi elaborada e analisada, o que possibilitou a verificação de que não houve relacionamentos lineares entre as variáveis (linearidade), nem a presença de singularidade e multicolinearidade. Em relação a fatorabilidade, o tamanho das correlações e a adequação da amostra foram analisados. Foram encontrados valores de correlação superiores a 0,30 em mais de 50\% dos casos, indicando uma matriz provavelmente fatorizável. O teste de KMO também foi realizado e obteve-se um valor de 0,70, considerado um bom índice de adequação da amostra.

A extração inicial de fatores foi feita mediante análise dos componentes principais. Foi realizado o tratamento pairwise para os casos omissos. A análise dos componentes principais sugeriu uma estrutura empírica com 3 componentes, que explicam, em conjunto, $52,02 \%$ da variância total das respostas dos participantes aos itens do questionário. Foi seguido o critério dos eigenvalues (valores próprios) dos fatores maiores ou iguais a 1 (um). Considerando a importância de cada fator, cada um deles deveria explicar no mínimo $3 \%$ da variância total. Dessa maneira, chegou-se ao valor máximo de 3 fatores a serem extraídos. Ao realizar a análise do scree plot, foi verificada a existência de 3 a 4 fatores. A análise paralela de Horn, por sua vez, confirmou uma estrutura com 3 fatores para este instrumento.

Para uma confirmação definitiva da estrutura fatorial do instrumento, foi realizada a PAF, com método de rotação oblíqua e tratamento pairwise para casos omissos. Decidiu-se incluir na escala apenas os itens com cargas fatoriais iguais ou superiores a 0,30. Tal análise 
permitiu a extração de 3 fatores que explicam, respectivamente, $27,41 \%, 14,13 \%$ e $12,94 \%$ da variância total das respostas aos itens do instrumento. Somados explicam, portanto, $54,50 \%$ da variância total. Após toda a revisão de literatura, a maneira pela qual os itens se agruparam e formaram fatores fez sentido teórico. A tabela 2 apresenta a estrutura empírica da escala, as cargas fatoriais, as comunalidades $\left(\mathrm{h}^{2}\right)$ dos itens, as médias e desvios-padrão, os índices de consistência interna, os valores próprios e percentuais de variância explicada de cada fator.

Tabela 2. Estrutura empírica da Escala de Estilos de Aprendizagem.

\begin{tabular}{|c|c|c|c|c|c|c|}
\hline \multirow{2}{*}{ Descrição dos itens } & \multicolumn{3}{|c|}{ Cargas fatoriais } & \multirow{2}{*}{$\mathbf{h}^{2}$} & \multirow{2}{*}{$\mathbf{x}$} & \multirow{2}{*}{ DP } \\
\hline & 1 & 2 & 3 & & & \\
\hline $\begin{array}{l}\text { 4. Realizar uma atividade paralela enquanto } \\
\text { estudo, como ouvir música, assistir televisão, } \\
\text { navegar na internet. }\end{array}$ & & & & 0,16 & 2,34 & 1,44 \\
\hline 10. Estudar juntamente com outros alunos. & & & 0,84 & 0,72 & 3,29 & 1,37 \\
\hline $\begin{array}{l}\text { 12. Participar das atividades de interação virtual } \\
\text { (fóruns, bate-papo) para discutir os conteúdos com } \\
\text { os outros estudantes. }\end{array}$ & & & 0,31 & 0,12 & 2,98 & 1,29 \\
\hline $\begin{array}{l}\text { 13. Receber instruções detalhadas sobre as } \\
\text { atividades a serem realizadas no curso. }\end{array}$ & & 0,39 & & 0,29 & 4,32 & 0,90 \\
\hline $\begin{array}{l}\text { 15. Que os professores/tutores expliquem } \\
\text { claramente o que é importante estudar para } \\
\text { alcançar os objetivos propostos no curso. }\end{array}$ & & 0,48 & & 0,38 & 4,08 & 1,05 \\
\hline $\begin{array}{l}\text { 20. Realizar meus estudos em lugares bem } \\
\text { iluminados. }\end{array}$ & 0,61 & & & 0,49 & 4,59 & 0,82 \\
\hline 21. Estudar em ambientes com pouco barulho. & 0,86 & & & 0,81 & 4,54 & 0,87 \\
\hline $\begin{array}{l}\text { 23. Que sejam realizadas avaliações de } \\
\text { aprendizagem para verificar meu domínio do } \\
\text { conteúdo apresentado. }\end{array}$ & & & & 0,09 & 3,84 & 1,02 \\
\hline 25. Ter o material impresso para ler. & & 0,44 & & 0,24 & 4,04 & 1,31 \\
\hline $\begin{array}{l}\text { 26. Ser lembrado sobre os prazos de entrega das } \\
\text { atividades do curso. }\end{array}$ & & 0,85 & & 0,70 & 4,41 & 0,97 \\
\hline $\mathbf{N}$ & 134 & 131 & 134 & & & \\
\hline Eigenvalue (valor próprio) & 2,74 & 1,41 & 1,29 & & & \\
\hline \% da variância explicada & 27,41 & 14,13 & 12,94 & & & \\
\hline $\mathbf{N}^{\circ}$ de itens & 2 & 4 & 2 & & & \\
\hline Alfa $(\alpha)$ & 0,76 & 0,63 & 0,40 & & & \\
\hline
\end{tabular}

O instrumento de Estilos de Aprendizagem possui 10 itens e é formado por 3 fatores que foram chamados de: Fator 1 "Ambiente de Estudo", Fator 2 "Regulação Externa" e Fator 3 "Interação Interpessoal". O item 23 "Que sejam realizadas avaliações de aprendizagem para verificar meu domínio do conteúdo apresentado" não permaneceu na estrutura por não possuir carga fatorial igual ou 
maior que 0,30, positiva ou negativa. O item 4 "Realizar uma atividade paralela enquanto estudo, como ouvir música, assistir televisão, navegar na internet" inicialmente foi incluso no fator 1 , denominado "Ambiente de Estudo". Porém, ao realizar o cálculo de confiabilidade, o valor do Alfa apresentou um valor negativo, e foi indicado que o mesmo teria seu valor alterado caso esse item fosse retirado do fator. Sendo assim, optou-se por sua exclusão do instrumento.

O Fator 1, "Ambiente de Estudo", apresenta um total de 2 itens e possui um bom índice de consistência interna $(a=0,76)$, com cargas fatoriais variando entre 0,61 e 0,86 . Os dois itens pertencentes a esse fator tratam de questões relativas ao ambiente no qual o estudo está sendo realizado, e as preferências do aluno em relação a esse contexto. São confirmados pela literatura em Zerbini (2007), que afirma que os Estilos de Aprendizagem também envolvem preferências relativas ao contexto de estudo do aluno.

O Fator 2, "Regulação Externa", apresenta um total de 4 itens e possui um moderado índice de consistência interna $(a=0,63)$, com cargas fatoriais variando entre 0,39 e 0,85 . Todos os itens pertencentes a esse fator foram escritos baseado no fator "Regulação Externa da Aprendizagem" do instrumento de Estilos Preferenciais de Aprendizagem de Brant (2014), que descreve preferências do aluno em ter sua aprendizagem regulada por fatores externos a ele, como por exemplo o material didático e/ou os professores/tutores.

O Fator 3, "Interação Interpessoal", é composto por 2 itens e apresenta um baixo índice de consistência interna $(a=0,40)$, com cargas fatoriais variando entre 0,31 e 0,84 . Os itens desse fator remetem à preferência do aluno em estudar e/ou realizar as atividades do curso sozinho ou com os demais alunos. Tal preferência é citada por Vermunt (1998), Zerbini (2007) e Brant (2014).

\section{Discussão e Considerações Finais}

Os resultados fazem com que o objetivo desta pesquisa seja cumprido, no sentido que constrói e verifica evidências de validade do instrumento de Estilos de Aprendizagem. $O$ instrumento final de Estilos de Aprendizagem, após passar pela revisão ficou com apenas 8 itens. $O$ instrumento deve receber novos itens, especialmente nos fatores Interação Interpessoal e Ambiente de Estudo que contam somente com dois itens cada um. Deve ser realizada a tentativa de inclusão de novos itens, para que seja verificado se o construto Estilos de Aprendizagem é contemplado somente por esses itens do instrumento ou pode haver outros itens que são abordados pela definição. É importante salientar que a inserção de novos itens deve respeitar a definição do construto. Em relação aos 10 itens finais 
obtidos após a revisão do instrumento e análise conceitual do construto, nenhum deles apresentou elevados índices de correlação e foram extraídos três fatores, a saber: Ambiente de Estudo, Regulação Externa e Interação I nterpessoal.

O item "... realizar individualmente as tarefas" não foi computado pelo Google Docs, ou seja, as respostas a essa questão não foram gravadas. Sendo assim, sugere-se que em uma próxima aplicação o item seja incluído novamente, pois, segundo a definição de Brant (2014) e Vermunt (1998), trata de um Estilo de Aprendizagem.

O item 23 "Que sejam realizadas avaliações de aprendizagem para verificar meu domínio do conteúdo apresentado" não permaneceu na estrutura por não possuir carga fatorial igual ou maior que 0,30. Tal item pertence ao fator "Regulação Externa da Aprendizagem" de Brant (2014), que define preferências do aluno em ter sua aprendizagem regulada pelo material didático, pelos professores, etc. Um possível motivo pelo qual o item não permaneceu no questionário nessa amostra é que a aplicação de avaliações não é uma preferência, pois ela ocorrerá inevitavelmente.

O item 4 "Realizar uma atividade paralela enquanto estudo, como ouvir música, assistir televisão, navegar na internet" inicialmente foi incluso no fator 1, denominado "Ambiente de Estudo". Porém, ao realizar o cálculo de confiabilidade, o valor do Alfa apresentou um valor negativo, e foi indicado que o mesmo teria seu valor alterado caso esse item fosse retirado do fator. Sendo assim, optou-se por sua exclusão do instrumento. Uma possível explicação para esse fato é que o item não trata de uma condição ambiental para o estudo, não demonstrando, portanto, relação com o fator em questão.

O primeiro fator, Ambiente de Estudo ( 2 itens, $a=0,76$, cargas fatoriais entre 0,61 e 0,86$)$. Os itens estão relacionados a definição de Zerbini (2007), que aponta como Estilos de Aprendizagem as preferências do aluno em relação ao ambiente no qual se realiza os estudos.

O fator número 2, Regulação Externa (4 itens, a=0,63, cargas fatoriais entre 0,39 e 0,85), apresentou itens pertencentes ao fator Regulação Externa de Brant (2014). Tal fator avalia, segundo a autora, as preferências relacionadas às estratégias e procedimentos instrucionais utilizados para orientação e controle do processo de aprendizagem.

Por fim, o fator 3, Interação Interpessoal ( 2 itens, $a=0,40$, cargas fatoriais entre 0,31 e 0,84 ), possui itens relacionados à preferência do aluno em estudar e/ou realizar as atividades do curso sozinho ou com os demais alunos. Tal fator está presente no instrumento de Brant (2014) com o nome de Interação, e no instrumento de Salles (2007) com o nome de Preferência Interpessoal. Além disso, essa preferência é citada por Vermunt (1998), Zerbini (2007) e Brant (2014). Sendo assim, apesar de apresentar um índice de consistência 
interna não satisfatório, foi decidido manter o fator, incluir mais itens em uma próxima aplicação e reavaliar sua validade.

Segundo Martins (2012), a área de avaliação de cursos no nível superior de ensino, especificamente, cursos de graduação ofertados a distância é bastante incipiente e carece de estudos sistemáticos, os quais proponham modelos multivariados de avaliação e façam uso de instrumentos de medida para cursos na modalidade EAD. Deve-se considerar que este é um campo de conhecimento ainda em fase de exploração, com poucas pesquisas e instrumentos disponíveis na literatura. Por esse motivo, recomenda-se que a escala utilizada nessa pesquisa seja aplicada em diferentes contextos de IES e amostras que estudem essencialmente a distância.

Ainda no contexto de EaD em IES, o estudo de Estilos de Aprendizagem é extremamente raro. Em outros contextos, destacase a falta de instrumentos fidedignos e válidos. Outro ponto é sua relação e diferenciação com o conceito de Estratégias de Aprendizagem de Zerbini (2007) e Warr e Allan (1998), apresentados anteriormente. Após a realização das análises fatoriais exploratórias do instrumento, obteve-se escalas psicometricamente válidas e confiáveis em sua maioria. Os próximos passos devem incluir Análises Fatoriais Confirmatórias do instrumento de Estilos de Aprendizagem. $O$ instrumento deve receber novos itens e ter alguns dos seus atuais itens reescritos. Outra possibilidade são os procedimentos de análises de dados mais robustos e rigorosos para o teste de relações entre variáveis de TD\&E, como a modelagem por equação estrutural (MEE). Tais procedimentos são recomendados por Zerbini (2007) e Martins (2012) e auxiliam no desenvolvimento do campo teórico da área de avaliação instrucional.

Dentre as contribuições deste estudo, destacam-se: (1) Revisão de literatura sobre a variável "Estilos de Aprendizagem" nos campos de conhecimento da Psicologia e da Educação; (2) Construção de um instrumento de Estilos de Aprendizagem no contexto de ações educacionais ofertadas a distância em IES, baseado no campo teórico da POT; (3) Verificação de evidências de validade do instrumento de "Estilos de Aprendizagem", que podem ser utilizados em futuras pesquisadas da área de avaliação de ações educacionais a distância, em O\&T e IES que ofertam cursos EAD.

Quanto às limitações do estudo, pode-se citar: (1) Não utilização de métodos estatísticos mais robustos, tais como a Análise Fatorial Confirmatória e Modelagem de Equação Estrutural, sendo que essas duas últimas poderiam colaborar na testagem de modelos de predição; (2) Impossibilidade de generalizar os resultados encontrados na presente pesquisa para outros contextos e participantes, pois foi avaliado apenas um curso, de apenas uma IES; (3) Uso de apenas uma fonte de informação: os alunos do curso participante da IES avaliada - deve-se, em estudos futuros, incluir 
outros atores envolvidos nas ações educacionais, como os tutores; (4) Instrumento de Estilos de Aprendizagem pouco consistente, com poucos itens e necessitado de uma revisão e melhorias.

\section{Referências}

Abbad, G. S., Carvalho, R. S., \& Zerbini, T. (2006). Evasão em curso via internet: explorando variáveis explicativas. Revista de Administração de Empresas Eletrônica, 5(2). Recuperado de http://www.scielo.br/pdf/raeel/v5n2/v5n2a08.pdf

Berings, M. G. M. C., Poell R. F., \& Simons, P. R. J. (2005). Conceptualizing on-the-job learning styles. Human Resource Development Review, 4, 373-400.

Bjork, R. A., Dunlosky, J., \& Kornell, N. (2013). Self-Regulated Learning: Beliefs, Techniques, and Illusions. Annual Review of Psychology, 64, 417-44.

Brant, S. R. C. (2014). Estudo multinível de antecedentes do impacto no trabalho e da persistência/Evasão em treinamentos a distância (Tese de doutorado). Instituto de Psicologia, Universidade de Brasília, Brasil.

Brusilovsky, P., \& Peylo, C. (2003). Adaptative ans inteligente Webbased educational systems [Special Issue]. International J ournal of Artificial Intelligence in Education, 13, 159-172.

Coelho Jr., F. A., \& Borges-Andrade, J. E. (2008). Uso do conceito de aprendizagem em estudos relacionados ao trabalho e organizações. Paidéia, 18(40), 221-234.

Coll, C., Mauri, T., \& Onrubia, J. (2010). A incorporação das tecnologias da informação e da comunicação na educação - do projeto pedagógico às práticas de uso. In C. Coll \& C. Monereo (Orgs.). Psicologia da Educação virtual - Aprender e ensinar com as tecnologias da informação e da comunicação (pp. 6693). Porto Alegre: Artmed.

Felder, R. M., \& Silverman, L. K. (1988). Learning and teaching styles in engineering education. Engineering Education, 78(7), 674681.

Honey, P. \& Mumford, A. (2000). The Learning Styles Questionnaire: 80 Item Version. Maidenhead, Berkshire, UK: Peter Honey Publications.

Kolb, A. Y., \& Kolb, D. A. (2005). The Kolb Learning style inventoryversion 3.1: 205 technical specifications. Boston, MA: Hay Resources Direct.

Kolb, D. A. (1985). Learning style inventory: self-scoring inventory and interpretation booklet. Boston, MA: McBer and Company.

Martins, L. B. (2012). Aprendizagem em ações educacionais a distância: fatores influentes no desempenho acadêmico de 
universitário. Dissertação de Mestrado, Faculdade de Filosofia, Ciências e Letras de Ribeirão Preto, Universidade de São Paulo, São Paulo, Brasil.

Nichols, D. P. (1999) My Coefficient a is Negative, SPSS Keywords, Number $68 . \quad$ Disponível em <http://www.ats.ucla.edu/STAT/SPSS/library/negalfa.htm>. Acessado em 04/08/2006.

Pasquali, L. (2004). Análise fatorial para pesquisadores. Petrópolis: Vozes.

Sales, P. A. O. (2009). Evasão em Cursos a Distância: Motivos Relacionados às Características do Curso, do Aluno e do Contexto de Estudo (Dissertação de Mestrado). Instituto de Psicologia, Universidade de Brasília, Brasília, Brasil.

Salles, T. J. (2007). Estilos de Aprendizagem no Trabalho: Análise e Construção de Medidas (Dissertação de Mestrado). Instituto de Psicologia, Universidade de Brasília, Brasília, Brasil.

Tabachnick, B. G., \& Fidell, L. S. (2007). Using multivariate statistics. New York: Harper-Collins College Publishers.

Umekawa, E. E. R. \& Zerbini, T. (2015). Evasão e persistência em ações educacionais a distância: análise do perfil discente. Revista Psicologia: Organizações e Trabalho, 15(2), 188-200.

Varanda, R. C., Zerbini, T., \& Abbad, A. (2010). Construção e Validação da Escala de Reações à Interface Gráfica para Cursos de Educação a Distância. Psicologia: Teoria e Pesquisa, 26(2), 371-380.

Vermunt, J. D. (1998). The regulation of constructive learning processes. British Journal of Education Psychology, 68, 149171.

Vermunt, J. D. (2005). Relations between student learrning patterns and personal and contextual factors and academic performance. Higher Education, 49(3), 205-234.

Walter, A. M. (2006). Variáveis Preditoras de Evasão em Cursos a Distância (Dissertação de Mestrado). Universidade de Brasília, Brasília, Brasil.

Warr, P., \& Allan, C. (1998). Learning strategies and occupational training. Internacional Review of Industrial and Organizational Psychology, 13, 83-121.

Zerbini, T. (2007). Avaliação da transferência de treinamento em curso a distância (Tese de Doutorado). Instituto de Psicologia, Universidade de Brasília, Brasil.

Zerbini, T. \& Abbad, G. (2008). Estratégias de Aprendizagem em Curso a Distância: Validação de uma Escala. Psico-USF, 13(2), 177-187. 


\section{Endereço para correspondência}

\section{Raíssa Bárbara Nunes Moraes}

Universidade de São Paulo

Faculdade de Filosofia, Ciências e Letras de Ribeirão Preto

Av. Bandeirantes, 3900, Monte Alegre, CEP 14040-901, Ribeirão Preto - SP, Brasil

Endereço eletrônico: raissa.nmoraes@gmail.com

\section{Thaís Zerbini}

Universidade de São Paulo

Faculdade de Filosofia, Ciências e Letras de Ribeirão Preto

Av. Bandeirantes, 3900, Monte Alegre, CEP 14040-901, Ribeirão Preto - SP, Brasil

Endereço eletrônico: thais.zerbini@gmail.com

Recebido em: 04/08/2016

Reformulado em: 23/06/2017

Aceito em: 18/07/2017

\section{Notas}

* Psicóloga, Doutoranda pelo Departamento de Psicologia da Faculdade de Filosofia, Ciências e Letras de Ribeirão Preto - Universidade de São Paulo.

** Doutora e mestre em Psicologia pela Universidade de Brasília, professora doutora em Psicologia Organizacional e do Trabalho da Faculdade de Filosofia, Ciências e Letras de Ribeirão Preto - Universidade de São Paulo.

Este artigo de revista Estudos e Pesquisas em Psicologia é licenciado sob uma Licença Creative Commons Atribuição-Não Comercial 3.0 Não Adaptada. 